\title{
Current reported and observed hypertension status, sodium intake practices and body composition of adults in Riyadh city, Saudi Arabia
}

\author{
M. M. Alkhalaf, C. A. Edwards and E. Combet \\ Human Nutrition, School of Medicine, College of Medical, Veterinary and Life Sciences (MVLS), New Lister \\ Building, Glasgow Royal Infirmary10-16 Alexandra Parade, Glasgow G31 2ER, UK
}

Socioeconomic status and lifestyle (including dietary habits) have dramatically changed in Saudi Arabia (SA) over the last few dec$\operatorname{ades}^{(1)}$. SA suffers from a high burden of non-communicable diseases such as obesity and hypertension (HTN) ${ }^{(1-2)}$. HTN is associated with several health issues such as cardiovascular disease, stroke and coronary heart diseases ${ }^{(3)}$. The aim of the present study was to assess the prevalence of HTN and related risk factors (RFs), and salt and sodium use in a random sample of residents in Riyadh, SA.

Participants aged 19-60 years were randomly selected at large retail centres in areas of contrasting socio-economic status in Riyadh, during October and November 2013. Informed consent was collected. Dietary patterns, including socio-demographics, lifestyle (smoking, physical activity), salt intake and health status using a validated questionnaire. Height, weight, waist circumference (WC) were measured. Systolic and diastolic blood pressures were measured twice within 20 minutes using an automatic sphygmomanometer. Hypertension was defined as systolic blood pressure $\geq 140$ or diastolic blood pressure $\geq 90 \mathrm{mmHg}$.

A total of 601 participants were recruited (265 males, 336 females, median age 29, IQR 24-38). Most participants (57.9\%) were educated to college level or higher, however, personal income was low $(<42,000 \mathrm{SR} /$ year, equivalent to minimum wage) for nearly half $(44 \cdot 1 \%)$, potentially due to the proportion of females no in paid occupation in SA.

\begin{tabular}{|c|c|c|c|c|c|c|}
\hline \multirow[b]{2}{*}{ Category } & \multicolumn{2}{|c|}{ Whole Population } & \multicolumn{2}{|c|}{ Without HTN * } & \multicolumn{2}{|l|}{ HTN* $^{*}$} \\
\hline & $n(\%)$ & $\overline{\text { median age }(I Q R)}$ & $\overline{n(\%)}$ & median age (IQR) & $\overline{n(\%)}$ & median age $(I Q R)$ \\
\hline $\mathrm{BMI} \leq 25$ & $200(33 \cdot 3)$ & $25(22-29)$ & $187(35 \cdot 7)$ & $25(22-29)$ & $12(6 \cdot 0)$ & $23(21 \cdot 5-25 \cdot 3)$ \\
\hline BMI 25-29.9 & $185(30 \cdot 8)$ & $30(24-39)$ & $160(30 \cdot 5)$ & $29(24-37)$ & $25(13 \cdot 5)$ & $45(33-51)$ \\
\hline BMI 30-39.9 & $191(31.8)$ & $35(27-45)$ & $160(30 \cdot 5)$ & $34.5(27-43)$ & $32(16 \cdot 8)$ & $40 \cdot 5(29 \cdot 8-50)$ \\
\hline $\mathrm{BMI} \geq 40$ & $24(4 \cdot 0)$ & $36(28-45 \cdot 5)$ & $17(3 \cdot 2)$ & $32(28-42)$ & $7 \cdot 0(28)$ & $44(31 \cdot 5-47 \cdot 5)$ \\
\hline Total & 600 & $29(23 \cdot 7-38)$ & 524 & $28(23-37)$ & 76 & $38.5(28-50)$ \\
\hline $\mathrm{WC}<80 \mathrm{~F},<94 \mathrm{M}$ & $278(46 \cdot 5)$ & $25(22--0)$ & $260(49 \cdot 8)$ & $25(22-30)$ & $18(6 \cdot 5)$ & $23 \cdot 5(22 \cdot 3-32)$ \\
\hline WC $80-88 \mathrm{~F}, 94-102 \mathrm{M}$ & $142(23 \cdot 7)$ & $32 \cdot 5(27-42)$ & $121(23 \cdot 2)$ & $32(26-40)$ & $21(15.44)$ & $39(31-50)$ \\
\hline $\mathrm{WC}>88 \mathrm{~F} ;>102 \mathrm{M}$ & $178(29 \cdot 8)$ & $36 \cdot 5(29-47)$ & $141(27)$ & $35(29-45)$ & $37(20 \cdot 8)$ & $44(35-51)$ \\
\hline Total & 598 & $29(23 \cdot 3-38)$ & 522 & $28(23-37)$ & 76 & $38 \cdot 5(28-50)$ \\
\hline
\end{tabular}

* Measured

Prevalence of overweight and obesity based on BMI was $66 \cdot 6 \%(30 \cdot 8 \%$ and $35.8 \%$, respectively). Over half of the population $(53.5 \%)$ had elevated waist circumference (females, $>80 \mathrm{~cm}$, males $>94 \mathrm{~cm}$ ). A total of $n=76(12 \cdot 6 \%)$ were hypertensive (median age 38, IQR 28-50). Of these, 44 (57.9\%) were females (median age 41, IQR 28-50) and 32 (42.1\%) males (median age 37, IQR 29-45). Only $31.6 \%$ of the hypertensive participants were aware that they had HTN. The occurrence of HTN was strongly associated with obesity. HTN increased proportionately with body mass index (BMI) and WC $(p<0.001)$. The median sodium intake for the total population was $4.0 \mathrm{~g} / \mathrm{d}$ (IQR $2.7-5.4 \mathrm{~g} / \mathrm{d}$ ) equivalent to $10 \mathrm{~g}$ salt/d (IQR $6.8-13.5 \mathrm{~g} / \mathrm{d}$ ), higher than recommended levels of $<2 \mathrm{~g} / \mathrm{d}$ for sodium ${ }^{(3)}$. Those reporting using salt substitutes described their use of mono-sodium glutamate (36\%) and sea salt $(35.7 \%)$, both high in sodium and not true substitutes. Population interventions, including health education, are required to manage the high prevalence of overweight and obesity, and reduce sodium intake in this population.

1. Khatib O (2004). East Mediterr Health J 10, 778-788.

2. Al-Nozha MM, Abdullah M, Arafah MR et al. (2007) Saudi Med J 28, 77-84.

3. WHO. (2003) WHO Global Strategy on Diet, Physical Activity and Health: Eastern Mediterranean Regional Consultation Meeting Report. Geneva. 